\title{
Measurement of current flow through the rat under signaled and unsignaled grid-shock conditions
}

\author{
HIROSHI IMADA, TETSURO MINO, KOZO SUGIOKA, and YUJI OHKI \\ $K$ wansei Gakuin University, Nishinomiya, Japan
}

\begin{abstract}
The present experiment was run to test the hypothesis that, when shock was signaled, rats would develop effective coping responses so as to reduce the current flow through them. A 1-sec shock was delivered through a grid floor by a fixed impedance ac shock source. The current-flow measure was taken over the last 30 of 90 trials given over 3 days and indexed by "gross skin conductance" or GSC (shock). The rat under the signaled shock condition $(n=15)$ showed higher GSC (shock) than did the rats under the unsignaled shock condition $(n=14)$. Thus, the result contradicted the hypothesis. There was no indication that the rats developed any preparatory response during the 5-sec signal, in terms of either GSC (signal) or posture. The results were discussed with reference to the preparatory-response hypothesis and various other possibilities.
\end{abstract}

A considerable number of rat experiments conducted both in the present writers' laboratory (Imada \& Okamura, 1975; Imada \& Soga, 1971; Nageishi \& Imada, 1974) and in other laboratories (e.g., Seligman, 1968; Seligman \& Meyer, 1970) have shown that signaled electric shocks are less stressful than unsignaled shocks. In conditioned suppression experiments, rats' basal rate of licking or of leverpressing was shown to be suppressed less when brief electric shocks were given with a warning signal than when they were given unsignaled. It is also known that animals choose a situation in which shocks are signaled rather than unsignaled. This phenomenon is generally known as PSS (preference for signaled shock) and was first shown by Lockard (1963). Since, in all the above studies, the shocks rats received were made exactly equal, both in intensity and duration, regardless of condition, the demonstrated difference in stress in rats under the signaled and unsignaled conditions has been ascribed to the psychological effect of "unpredictability" of shock.

Fanselow (1980) has recently summarized the major hypotheses that have been put forward to account for the above facts: the information hypothesis (Berlyne, 1960), the preparatory-response hypothesis (Perkins, 1968), the safety-signal hypothesis (Seligman, 1968), the time-allocation hypothesis (Rachlin, 1976), and a new hypothesis based on Pavlovian condition-

This research was supported by a Grant in Aid for Scientific Researches (No. 245016), Ministry of Education of Japan, to Hiroshi Imada. The authors are grateful for the assistance of Hajime Ninohira and Akihiko Yamazaki in running the experiment. $\mathrm{K}$. Sugioka is now at Department of Anatomy, Shimane Medical University, Izumo, Japan. ing principles. Although the present writers have turned mainly to the safety-signal hypothesis to account for their findings so far, the present experiment was conducted with special reference to the preparatory-response hypothesis.

To quote from Fanselow (1980, p. 66), "the preparatory-response hypothesis states that the signal allows the animal to respond in such a way that the reduction in aversiveness serves to reinforce this preparatory response (Perkins, 1968) .... There are a number of possibilities for the way the animal might prepare itself for shock, the most obvious of which is that the signal allows accurate timing of a postural adjustment that reduces the current flowing through the animal." The present experiment was designed to test the adequacy of this possibility by measuring the current flowing through a rat during grid shocks delivered by a fixed-impedance ac shock generator in order to determine if rats would develop any peripheral coping responses to reduce the aversiveness of shocks under the signaled shock condition.

Several characteristics of the present experiment should be mentioned to make the intention of the study clear. The experiment was conducted in a small box in which brief scrambled grid shocks were administered over 90 trials. The size and structure of the box and the intensity and duration of both the shock and the signal were made approximately the same as those used in the conditioned suppression studies, conducted in the present writers' laboratory, which have evidenced the stress-reducing effect of the signal (e.g., Imada \& Okamura, 1975; Nageishi \& Imada, 1974). It should be noted that this effect appeared even after a single session of six trials (see Figure 2, Imada \& Okamura, 1975). If the preparatory-response 
hypothesis had anything to supplement the safetysignal hypothesis in accounting for the function of the signal in a shock situation, the difference in the current flow through the rats under the two conditions should become evident in the present setting.

In an experiment similar in concept to the present one, Furedy and Biederman (1967), using an inkpen recorder, measured the current flow through the rat in a PSS situation. They proposed that rats develop a coping response to reduce current flow. But their experiment was run in a relatively large box using unscrambled 5-sec grid shock, which is unusually long for PSS studies. When the shock is long and unscrambled, there is more opportunity for the rat to find a safe pair of grid bars, so the results they obtained are not very surprising. The present experiment eliminated these problems.

\section{METHOD}

\section{Subjects}

The subjects were 29 male albino rats of the Wistar strain; they were divided into a signaled shock group (Group Sig; $n=15$ ) and an unsignaled shock group (Group Unsig; $n=14$ ). The means of the body weights of the two groups were $412.7 \mathrm{~g}$ (range, 333-492 g) and $411.3 \mathrm{~g}$ (range, $343-498 \mathrm{~g}$ ), respectively.'

\section{Apparatus}

The shockbox used was made of brown Bakelite plates and transparent acrylic plates, and the inside dimensions were $20 \times 10$ $\times 15 \mathrm{~cm}$ (height). The floor of the box consisted of copper rods, $3 \mathrm{~mm}$ in diameter and separated from each other by $13 \mathrm{~mm}$, measured from center to center. It should be noted that, because of the smallness of the apparatus, escape from shock by jumping up in the air was virtually impossible. Also, because of the narrowness of the box, it was impossible for the rat to orient itself and sit in parallel with the grid bars. The signal was a tone
$[1,000 \mathrm{~Hz}, 85 \mathrm{~dB}(\mathrm{c})]$ presented through a loudspeaker suspended $195 \mathrm{~cm}$ above the ceiling of the shock box. The source of illumination was a minibulb suspended $31 \mathrm{~cm}$ above the ceiling of the box.

The shock generator was of an ordinary ac type, as shown in Figure 1. A total of $250-\mathrm{k} \Omega$ current-limiting resistance was put in series with the rat, and 1-sec shock of $150 \mathrm{~V}$, measured on the secondary side of the transformer, was given through a scrambler. The current flow through a rat was indexed by using a measure designated "gross skin conductance" (GSC). When the switch was thrown into B position (Figure 1), the electric shock could be applied to the rat. At the same time, the voltage drop across $\mathrm{X}$ and $Y$ was recorded. The voltage drop across $X$ and $Y$, of course, changes in accordance with the rat's movements and/or changes in its skin resistance, and they are reflected in moment-to-moment changes in the height of 120 peaks of $60-\mathrm{Hz}$ alternating current. These changes were recorded in the data recorder and later reproduced and converted into digital values through an $A / D$ convertor of a minicomputer, PDP-11/10(AR 11), which provided the GSC measure called GSC (shock). ${ }^{2}$ With the switch in position A (Figure 1), a weak shock of $1 \mathrm{sec}$, to which a rat was not sensitive, could also be applied during signals and during ITIs, and the GSC (signal) and GSC (base) measures, respectively, were taken by measuring the voltage drop across $\mathrm{X}$ and $\mathrm{Z}$ of Figure 1 (see also Figure 2).

\section{Procedure}

Rats were placed individually into the shock box and, after $1 \mathrm{~min}$, the first shock was given. On each trial, $150-\mathrm{V}$ shock of $1 \mathrm{sec}$ duration was given through the grid floor. The only difference between Group Sig and Group Unsig was the temporal positions at which the signals were presented. For Group Sig, the signal of 5 sec duration was invariably given immediately preceding each shock onset, as shown in Figure 2. For Group Unsig, the signals were given independently of shocks. The signals for this group were presented once per ITI, preceding shocks by a mean of $26.8 \mathrm{sec}$ (range, $10-45 \mathrm{sec}$ ). The experiment was continued for 3 successive days, and 30 trials were given per day with a mean intershock interval of $60 \mathrm{sec}$ (range, 30-90 sec). Complete data were taken only on Day 3 for Trials 61-90.

The primary measures of the present study were gross skin con-

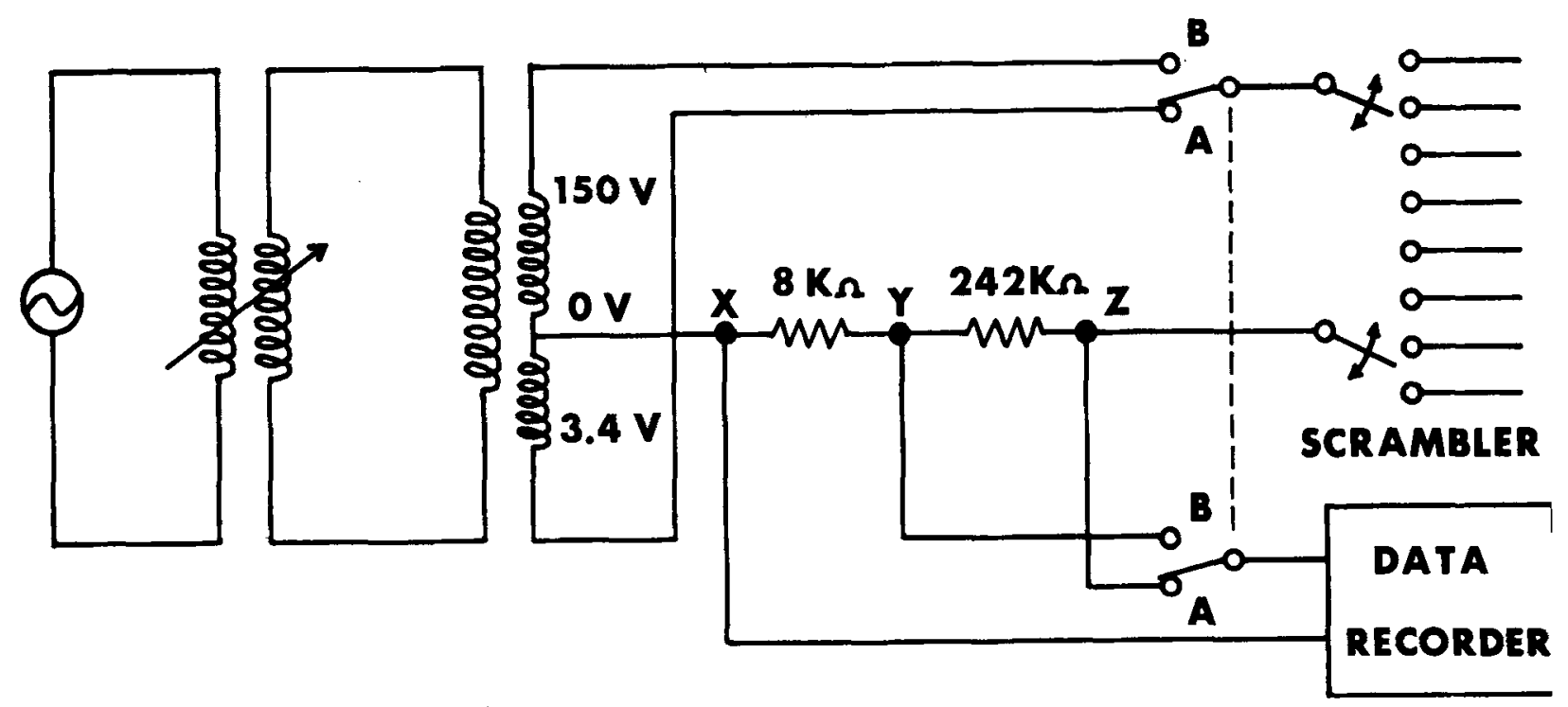

Figure 1. Circuit diagram of the shock generator and the device for measuring GSCs. 


\section{TIME (SEC)}

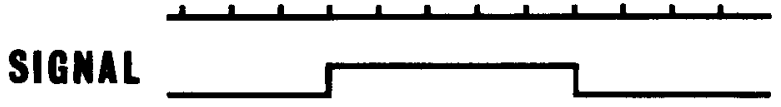

SHOCK

GSC

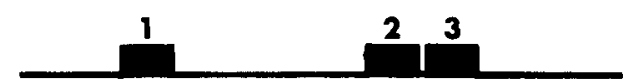

1.GSC (BASE) 2.GSC(SIGNAL) 3.GSC (SHOCK)

Figure 2. Signal-shock relationship of Group Sig and temporal positions where GSC measures were taken.

ductance measured at different times: GSC (shock), GSC (signal), and GSC (base). ${ }^{3}$ As shown in Figure 2, the GSC (signal) and GSC (base) were measured for Group Sig for $1 \mathrm{sec}$ starting $1.2 \mathrm{sec}$ before shock onset and signal onset, respectively. For Group Unsig, only data for GSC (shock) and GSC (base) were collected. During each 1-sec shock, the current flow during each of the 120 voltage maxima was recorded by the computer, and the median and the interquartile range were computed. These median currents were the basic trial data. The GSCs of 150 and 128 approximately correspond to the resistance values of 50 and $100 \mathrm{k} \Omega$, respectively, as shown by the reference resistance level (RRL) along the right ordinate of Figure 3.

The number of feet with which the rat touched the grid floor at the moment of shock onset was carefully observed through colored cellophane attached to the transparent front wall of the box.

\section{RESULTS}

The left half of Figure 3 represents the means of median GSC (shock) of Trials 61-90 in blocks of five trials for Group Sig and Group Unsig. The GSC (shock) increased gradually over trials, and this tendency is more remarkable in Group Sig than in Group Unsig. The results of a 2 (groups) by 3 (10-trial blocks) analysis of variance showed the main effect of trial blocks to be significant $[F(2,54)=9.34, p<$ $.001]$. But the main effect of groups and the interaction effect of the two terms were not significant $[F(1,27)=1.65$ and $F(2,54)=1.80$, respectively $]$. Independent $t$ tests for the last five trials revealed that the difference was significant at approximately $\mathrm{p}=.05(\mathrm{t}=2.01)$. The means of the interquartile range, an index of the variability of responses to shock, were, in successive blocks of five trials, 25.0, 22.4, 26.1, 28.4, 24.7, and 21.9 for Group Sig and 24.0, 18.6, 19.3, 21.9, 19.9, and 21.4, for Group Unsig. An overall $t$ test between these mean $Q$ values was not significant $(\mathrm{t}=1.04)$.

The results regarding GSC (base) for Trials 61-90 are shown by the pair of dotted lines in the right half of Figure 3. The GSC (base) of both groups increased over trials, and the result of a 2 by 3 analysis of variance conducted in the same way as above revealed that only the main effect of trial blocks was significant $[F(2,54)=3.48, p<.05]$. The $F$ values for the other effects were $>1$.

The results of GSC (signal) for Group Sig are also shown on the right side of Figure 3. The data for this measure show essentially the same pattern and level as those for GSC (base) of the same group. The overall difference of GSC (base) and GSC (signal) was not significant $[\mathrm{t}(14)=1.13]$.

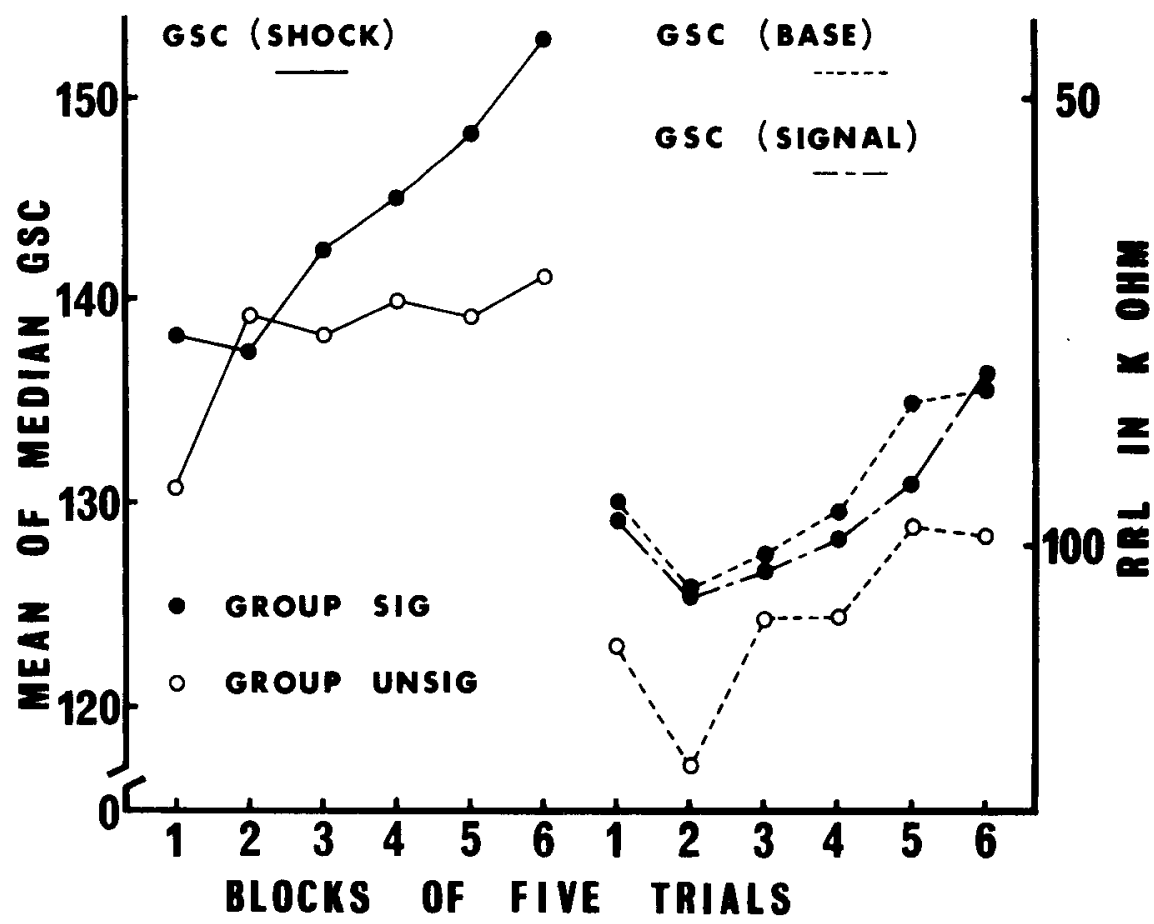

Figure 3. Means of median GSC (shock) (left half) and of median GSC (base) and GSC (signal) (right half) in blocks of five trials. 
The means of the number of the rat's feet touching the grid floor at the moment of shock onset were, in successive blocks of 10 trials, $3.47,3.19$, and 3.14 for Group Sig and 3.52, 3.46, and 3.32 for Group Unsig. The result of a 2 by $3 \mathrm{~F}$ test revealed all the obtained $F$ ratios to be $<1$.

\section{DISCUSSION}

If one assumes that "the aversiveness of shock stimulation is proportional to the current flowing through the rat"' (Campbell \& Masterson, 1969, p. 27) and, further, that GSC (shock) is an appropriate measure of the amount of current flow, then the present results regarding GSC (shock) are quite contradictory to what would be expected from the preparatory-response hypothesis. Group Sig rats seemed to have prepared in seemingly disadvantageous ways for the shock at the final stage of training so that, compared with Group Unsig rats, they allowed more current to flow through their bodies. It should be noted, in this connection, that Badia and Abbott (1980) have recently found no differential modification of shock contact time between rats in signaled and unsignaled shock conditions. The present result appears to be consistent with their findings.

How then should the present results be explained? One possibility is that something like GSR conditioning might have taken place in Group Sig rats, and they might have come to wait for the coming shock by increasing their skin conductance level before the shock presentation. This possibility, however, is immediately rejected in light of the fact of no difference between GSC (base) and GSC (signal) shown in Figure 3. Not only did Group Sig show no anticipatory increase in GSC to the signal, but also their posture, in terms of the number of feet touching the grid floor at the moment of shock onset, did not differ from that of Group Unsig rats. The finding of greater GSC (shock) of Group Sig over Group Unsig toward the end of the session, then, seems to suggest that Group Sig rats had made some preparation before the shock came on, which was not manifested in the measures taken during the signal.

A second possibility would be to deny that the current flow is a suitable index of the aversiveness of shock stimulation. Marlin, Berk, and Miller (1978, p. 204) have pointed out that "perceived aversiveness of a given shock is related to the total current flow through the subject, the current density, and the anatomical site of the shock." On the basis of their data, and because they put such a high impedance (2.5 M $\Omega$ ) in series with the rat, Marlin et al. emphasized the current density and the site as the important determinants of perceived aversiveness of a shock. On the basis of the present findings, it is quite possible to conclude, along with Marlin et al., that cur- rent flow is not an adequate measure of the perceived aversiveness of a shock. But, in order for this view to be supported more positively, one would have to show that the rats' gain, in terms of the current density and the anatomical site of the shock, is actually achieved even though there is a disadvantage in terms of current flow.

The third possibility is that rats' innate defense reactions, including the changes in the gross skin conductance and/or posture, which obviously should have survival values in the natural environment, are those which may bring disadvantage to them in such an unnatural or artificial environment as one with an electrified grid floor. This possibility argues against the view held by Marlin et al. (1978) described above. It is a challenge to those interested in the psychology of learning to find out if rats, which to begin with have many biological constraints, including ones that seem to humans to be disadvantageous, would, after thousands of trials in an environment that was unnatural to them, develop any coping response that appeared to be effective even to the experimenters. In other words, would it be possible for rats to be free of their biological constraints after a prolonged period of being trained in an unnatural environment? The present technique could be used to answer such a question.

Why, next, did the GSC measures shown in Figure 3 increase over trials? Anderson, Plant, and Paden (1967) and Kaplan (1963) have shown that rats' basal skin resistance decreased in stressful situations and that the amount of decrease tended to be greater when stronger shock was used than when weaker shock was used. Kaplan (1963) also showed that the rats' skin resistance in a nonshock situation increased gradually as the rats adapted to and became familiar with the situation. The present finding of increased GSC over trials could be explained with reference to these facts; that is, the stress produced by repeated presentation of shock could have led to decreased skin resistance or to increased GSC. It is also possible to argue that the increase in GSC over time may have been due to the increased amounts of urine on the grids and animals' feet over time during the session. Although nothing definite can be said regarding the nature of this possibility, it should be noted that this problem may also have to do with the point described in the paragraph immediately above.

The most conservative conclusion one can draw from the present findings would be that the rats in the signaled-shock condition did not develop any response to reduce the current flow through them. If the GSC (shock) is an adequate measure of rat's preparatory response, the preparatory-response hypothesis could not account for the experimental finding of stress-reducing effects of a signal in the conditioned suppression situation in a much smaller number of 
trials. But it should be noted, finally, that the results of the present experiment do not necessarily mean that rats would not develop effective coping attempts if (a) a larger number of trials were to be given and/or (b) other peripheral preparatory-response measures were taken. With regard to the former point, in a study undertaken in the present writers' laboratory (Imada, Shimai, \& Imada, in press), three of four rats placed in a rearing box (Shishimi \& Imada, 1977) and given 300-msec footshock with an FT 5-sec schedule 720 times per day over 55 days gradually developed, first, rearing responses and then jumping responses to shock. These responses were anticipatory in nature but did not appear to have obvious instrumental meaning. The fourth rat on Day 16 suddenly developed the jumping response, which effectively reduced the shock-contact time from $300 \mathrm{msec}$ to approximately $180 \mathrm{msec}$. Further attempts need to be made to understand the "wisdom" of rats in the grid-shock situation.

\section{REFERENCES}

Anderson, D. C., Plant, C., \& Paden, P. Conditioned suppression of a running response as related to competing responses, drive, and basal skin resistance level. Journal of Comparative and Physiological Psychology, 1967, 63, 282-287.

Badia, P., \& Aввott, B. Does shock modifiability contribute to preference for signaled shock? Animal Learning \& Behavior, $1980,8,110-115$.

Berlyne, D. E. Conflict, arousal and curiosity. New York: McGraw-Hill, 1960.

Campbell, B. A., \& Masterson, F. A. Psychophysics of punishment. In B. A. Campbell \& R. M. Church (Eds.), Punishment and aversive behavior. New York: Appleton-Century-Crofts, 1969.

Fanselow, M. S. Signaled shock-free periods and preference for signaled shock. Journal of Experimental Psychology: Animal Behavior Processes, 1980, 6, 65-80.

Furedy, J. J., \& Btederman, G. B. Preference for signaled shock phenomenon: Direct and indirect evidence for modifiability factors in the shuttlebox. Animal Learning \& Behavior, 1976, 4, 1-5.

Imada, H., \& OKamura, M. Some cues mats can use as predictors of danger and safety. Animal Learning \& Behavior, 1975, 3, 221-225.

Imada, S., Shimai, S., \& Imada, H. Behavior of rats under fixed-time shock schedule. Japanese Psychological Research, in press.

ImADA, H., \& SogA, M. The CER and BEL as a function of predictability and escapability of an electric shock. Japanese Psychological Research, 1971, 13, 115-122.

KaPLAN, R. Rat basal resistance level under stress and nonstress conditions. Journal of Comparative and Physiological Psychology, 1963, 56, 775-777.
LOCKARD, J. S. Choice of a warning signal or no warning signal in an unavoidable shock situation. Journal of Comparative and Physiological Psychology, 1963, 56, 526-530.

Marlin, N. A., Berk, A. M., \& Miller, R. R. Modification and avoidance of unmodifiable and unavoidable footshock. Bulletin of the Psychonomic Society, 1978, 11, 203-205.

NAGEISHI, Y., \& ImAdA, H. Suppression of licking behavior in rats as a function of predictability of shock and probability of conditioned-stimulus-shock pairings. Journal of Comparative and Physiological Psychology, 1974, 87, 82-87.

Perkins, C. C., JR. An analysis of the concept of reinforcement. Psychological Review, 1968, 75, 155-172.

RAChlin, H. Behavior and learning. San Francisco: Freeman, 1976.

Seligman, M. E. P. Chronic fear produced by unpredictable electric shock. Journal of Comparative and Physiological Psychology, 1968, 66, 402-411.

Seligman, M. E. P., \& Meyer, B. Chronic fear and ulcers in rats as a function of unpredictability of safety. Journal of Comparative and Physiological Psychology, 1970, 73, 202-207.

Shishim 1, A., \& Imada, H. Discriminated and nondiscriminated avoidance conditioning of the rearing response in rats. Animal Learning \& Behavior, 1977, 5, 259-264.

\section{NOTES}

1. The present experiment was originally conducted as part of a larger experiment and, before the present experiment was run, the subjects had been tested in an open field for 4 consecutive days, 2 min per day. All the subjects had also been tested to determine the absolute threshold of shock. The test used the method of limits; it consisted of the administration of 10 ascending series with a step of $10 \mu \mathrm{A}$. Each series was discontinued when a rat showed visually detectable movements in response to a $.5-\mathrm{sec}$ grid shock three times in succession.

2. The data system was calibrated with different resistors inserted in place of the rat. The results of this calibration indicated that the reported numerical values represent approximately 45 times the combined conductance (in micromhos) of the rat together with the series resistors. Thus, it is possible to calculate the rat's approximate resistance from a given numerical value of GSC by the formula $R=45 / G S C-.25$ (in megohms).

3. It should be noted that measures other than those described below were also taken. The shock box was actually mounted on a stabilimeter device and shock-elicited activity was measured and analyzed by the computer. But, since this device appeared to be sensitive only to vertical movements of the rat and not to horizontal movements, the activity thus measured could not be regarded as a suitable index of the vigor of rats' response to shocks. To avoid complication of the description, this part of the data was omitted from the present analysis. The activity measure did not show significant difference between the two groups of the present experiment.

(Received for publication April 1, 1980; revision accepted October 9,1980 .) 